\title{
PLK1 inhibition in cancer therapy: potentials and challenges
}

\author{
Inas Elsayed ${ }^{1,2,3,4}$ \& Xiaosheng Wang*,1,2,3 \\ ${ }^{1}$ Biomedical Informatics Research Lab, School of Basic Medicine \& Clinical Pharmacy, Nanjing 211198, PR China \\ ${ }^{2}$ Cancer Genomics Research Center, School of Basic Medicine \& Clinical Pharmacy, Nanjing 211198, PR China \\ ${ }^{3}$ Big Data Research Institute, China Pharmaceutical University, Nanjing 211198, PR China \\ ${ }^{4}$ Department of Pharmacology, Faculty of Pharmacy, University of Gezira, Wad Madani 20, Sudan \\ *Author for correspondence: xiaosheng.wang@cpu.edu.cn
}

'To highlight markers for sensitivity of cancer cells to PLK1 inhibitors, an in-depth understanding of PLK1 molecular circuit is important"”

First draft submitted: 22 March 2019; Accepted for publication: 26 March 2019; Published online: 12 July 2019

Keywords: cancer therapy • PLK1 • PLK1 inhibitors

\section{Background}

The last few decades have witnessed an evolving interest in polo-like kinase 1 (PLK1) inhibitors as anticancer drugs [1]. Such interest was warranted by the overexpression of PLK1 in a broad range of cancers and its association with the poor prognosis of cancer, as well as the improved safety profile of PLK1 inhibitors versus classic mitotic inhibitors [2,3]. However, only limited successes were reported by preclinical studies of PLK1 inhibitors up to now. In fact, several challenges are restraining the clinical utility of PLK1 inhibitors. First, most of the PLK1 inhibitors achieved therapeutic effects only when doses exceeded toxic limits. Second, the responses to PLK1 inhibitors reported from different studies were discrepant. Finally, the emergence of drug resistance is a major challenge $[1,3]$. Therefore overcoming these challenges represents a hot topic in current drug discovery research [4].

\section{Designing PLK1 inhibitors}

First of all, research should address how to foster the potency of PLK1-targeting drugs to avoid drug toxicity. This could be attained through developing probes with higher affinity and enhanced accessibility to PLK1 molecules. Concordantly, improving specificity of PLK1 inhibitors is equally important as it can reduce the therapeutic doses required via confining the drug to the pursued target. Furthermore, higher specificity can minimize the off-target toxicities. It is worth to note that specific PLK1 inhibition in cancer therapy is complicated by the structural conservation of kinase enzymes [3]. In addition, PLK1 plays multiple roles that vary with the cell cycle time and the cellular location [2]. Hence, pharmacological inhibition of this kinase should be designed to precisely block the specific function of PLK1 without affecting other unrelated functions. To do so, selecting the optimum target at PLK1 is crucial in determining the degree of specificity a developed PLK1 inhibitor can reach.

The first-generation PLK1 inhibitors were designed to target the catalytic N-terminal kinase domain (KD). Despite their ability to inhibit the viability and progression of cancer cells, effectiveness of such agents is markedly limited by the reduced selectivity toward PLK1. The conserved regions shared between the KDs of functionally distinct PLK subtypes as well as other protein kinases, increase the possibility of off-target adverse effects created by KD-binding PLK1 inhibitors. In addition, the emergence of drug resistance due to frequent mutations at the ATP-binding pocket could abort the therapeutic utility of such agents. To this end, investment has been taken into research to enhance drug selectivity through adopting the strategy of targeting the $\mathrm{KD}$ residues unique to PLK1 [3]. Another approach is to develop drugs that can bind to the inactive form of PLK1, which exposes less conserved residues of the molecule otherwise buried in the catalytically active conformation [3].

Despite the efforts to enhance the specificity of $\mathrm{KD}$ inhibitors, studies have shown that the kinase cross-reactivity observed in some less specific PLK1 inhibitors such as rigosertib, a non-ATP-competitive inhibitor, can augment the therapeutic efficacy. This indicates that the design of multitarget drugs modulating both PLK1 pathway and 
other complementary oncogenic pathways could be a promising strategy. In fact, studies have already evidenced the feasibility of developing such compounds [5]. However, the design of such drugs is a considerable challenge in that the activity of drugs must be tailored to precisely block PLK1 oncogenic effects, while exerting minimal effects on other functionally unrelated pathways. Once designed, however, such drugs are anticipated not only to enhance therapeutic efficacy, but also to minimize the chances for the development of drug resistance.

Alternatively, targeting the polo-like kinase box domain (PBD), which regulates enzyme's subcellular localization, substrate binding and kinase activity, is another viable choice. Because PBD is unique to the PLK family and has less sequence conservation compared with $\mathrm{KD}$, targeting PBD is expected to exhibit higher selectivity to PLK1 [3]. In fact, a number of small molecules, oligopeptides and natural compounds displaying PBD-binding capacity have been examined for their anticancer activity. Pharmacological utility of many of these compounds was hampered either by low potency, reduced cellular permeability and/or off-target reactivity [6]. Nevertheless, a few small chemical molecules, such as poloxin and its derivatives, have demonstrated reasonable activity in inhibiting cancer cell growth and replication [7]. Again, further research is needed to explore the efficacy and safety of such molecules in vivo and clinically.

\section{Combination of PLK1 inhibitors with other anticancer drugs}

Selecting the optimum target for design of PLK1 inhibitors may help overcome limited drug specificity and drug resistance. However, mitotic slippage and endo-reduplication represent another challenge associated with PLK1 inhibition consequences. Through such processes malignant cells may escape PLK1 inhibition-induced mitotic arrest culminating in a polyploid cell population. This will not only limit the anticancer efficacy of PLK1 inhibitors, but also lead to the generation of drug-resistant colonies. Furthermore, such polyploid colonies may retain malignant proliferative potentials due to genomic instability. The roles of a number of oncogenic pathways have been underscored in the study of potential mechanisms behind such events $[8,9]$. Hence, the combination of PLK1 inhibitors with other agents targeting the implicated pathways could be an effective tactic against such complications.

The combination of PLK1 inhibitors with other anticancer drugs has already been proved to be beneficial over monotherapy in clinical studies [1,10]. PLK1 inhibition may enhance the sensitivity of tumors to conventional chemo/radiotherapies, for example, the improved outcomes in acute myeloid leukemia treated with the combination of volasertib with low dose cytarabine [11]. However, to design the most effective PLK1 inhibitors-based combination therapy, we need to gain profound insights into the PLK1-mediated oncogenic mechanisms. Understanding the PLK1-regulatory network and its interactions with other oncogenic pathways is critical to pinpoint the key elements/pathways, which can be modulated to maximally synergize with PLK1 blockade. So far, a limited yet a noteworthy progress has been achieved in this area. For example, the detection of PI3K implication in mitotic slippage following PLK1 inhibition merited the combination of PLK1 inhibitors with PI3k inhibitors in cancer therapy. The combination has demonstrated promising results in preclinical studies against anaplastic thyroid tumors, a tenacious tumor otherwise having a poor prognosis [9]. Another successful combination was the use of PLK1 inhibitors with BCL-2 inhibitors, evidenced by the PLK1-mediated stabilization of the MYC oncogene in cancers [12].

Moreover, a recent study revealed a significant correlation between the high PLK1 expression and the antitumor immunity downregulation [13]. To explain such observation, several plausible justifications have been proposed. One justification is: because the cell cycle activity tends to suppress antitumor immunity, PLK1 inhibition may enhance antitumor immune response through inhibition of the cell cycle activity [13,14]. Another explanation is: the p53mediated enhancement of the MHC class 1 expression could be blocked by PLK1 activity [13,15]. Moreover, in vitro experiments have already demonstrated the effect of PLK1 inhibition in promoting tumor antigen presentation [13]. This observation rationalizes the combination of PLK1 inhibitors with cancer immunotherapy, although further experiments are needed to support such hypothesis [13].

\section{Markers for sensitivity to PLK1 inhibition}

To highlight markers for sensitivity of cancer cells to PLK1 inhibitors, an in-depth understanding of PLK1 molecular circuit is important. The inconsistent therapeutic responses to volasertib reported from different studies suggest that PLK1 inhibition may not be propitious to all cancers. In line with this, several studies have claimed the TP53 mutation is a biomarker for cancer sensitivity to PLK1 inhibition, indicating a reduced sensitivity of cancer cells expressing wild-type TP53 to PLK1 inhibition compared with TP53-mutant cells [16,17]. This suggests a 
compensatory mechanism mediated by $\mathrm{p} 53$ that rescues cancer cells from mitotic arrest and subsequent apoptosis caused by the PLK1 suppression [8]. Certainly, further research is needed to investigate the nature and components of such protective mechanism. Another potential marker for sensitivity to the PLK1 inhibition therapy is the RAS mutation status. It has been shown that cancers with activated KRAS are more addicted to PLK1 activity compared with those which lack KRAS activity, suggesting that KRAS-mutated malignancies are more sensitive to PLK1 inhibition than KRAS wild-type cancers [18]. However, experimental data on this issue collectively appeared to contradict. The conflicting results indicate that the PLK1 inhibition response is actually associated with the RAS pathway rather than the specific RAS protein [19]. This indicates that further research is needed to dissect the specific elements of the RAS pathway which direct the correlation with the sensitivity to PLK1 inhibition.

\section{Conclusion}

PLK1 inhibition holds potentials for cancer therapy while it is challenging for the development of effective PLK1 inhibitors in clinical cancer therapy. The in-depth analysis of PLK1 mechanisms and its interactions with other molecules and pathways is crucial for reaching an effective PLK1-modulating therapy with maximum potency, specificity, stability and optimal pharmacokinetic profile.

\section{Financial \& competing interests disclosure}

The authors have no relevant affiliations or financial involvement with any organization or entity with a financial interest in or financial conflict with the subject matter or materials discussed in the manuscript. This includes employment, consultancies, honoraria, stock ownership or options, expert testimony, grants or patents received or pending, or royalties.

No writing assistance was utilized in the production of this manuscript.

\section{References}

1. Gutteridge RE, Ndiaye M, Liu X, Ahmad N. PLK1 inhibitors in cancer therapy: from laboratory to clinics. Mol. Cancer Ther. 15(7), 1427-1435 (2016).

2. Liu Z, Sun Q, Wang X. PLK1, a potential target for cancer therapy. Transl. Oncol. 10(1), 22-32 (2017).

3. Chen JE, Glover GH. Recent advances and new strategies in targeting PLK1 for anticancer therapy. Trends Pharmacol. Sci. 36(12), 858-877 (2015).

4. Pearson RJ, Blake DG, Mezna M, Fischer PM, Westwood NJ, McInnes C. The Meisenheimer complex as a paradigm in drug discovery: reversible covalent inhibition through C67 of the ATP binding site of PLK1. Cell Chem. Biol. 25(9), 1107.e4-1116.e4 (2018).

5. Dietrich P, Freese K, Mahli A et al. Combined effects of PLK1 and RAS in hepatocellular carcinoma reveal rigosertib as promising novel therapeutic "dual-hit" option. Oncotarget 9(3), 3605-3618 (2018).

6. Archambault V, Normandin K. Several inhibitors of the PLK1 polo-box domain turn out to be non-specific protein alkylators. Cell Cycle 16(12), 1220-1224 (2017).

7. Klaeger S, Gande S, Kurunci-Csacsko E et al. Optimized PLK1 PBD inhibitors based on poloxin induce mitotic arrest and apoptosis in tumor cells. ACS Chem. Biol. 10(11), 2570-2579 (2015).

8. Smith L, Farzan R, Ali S et al. The responses of cancer cells to PLK1 inhibitors reveal a novel protective role for p53 in maintaining centrosome separation. Sci. Rep. 7(1), 1-12 (2017).

9. De Martino D, Yilmaz E, Orlacchio A et al. PI3K blockage synergizes with PLK1 inhibition preventing endoreduplication and enhancing apoptosis in anaplastic thyroid cancer. Cancer Lett. 439, 56-65 (2018).

10. Li J, Wang R, Schweickert PG et al. PLK1 inhibition enhances the efficacy of gemcitabine in human pancreatic cancer. Cell Cycle 15(5), 711-719 (2016).

11. Gjertsen BT, Schöffski P. Discovery and development of the Polo-like kinase inhibitor volasertib in cancer therapy. Leukemia 29(1), 11-19 (2015).

12. Ren Y, Bi C, Zhao X et al.PLK1 stabilizes a MYC-dependent kinase network in aggressive B cell lymphomas. J. Clin. Invest. 128(12), 5517-5530 (2018).

13. Li M, Liu Z, Wang X. Exploration of the combination of PLK1 inhibition with immunotherapy in cancer treatment. J. Oncol. 2018, 3979527 (2018).

14. Goel S, DeCristo MJ, Hoog J et al. CDK4/6 inhibition triggers anti-tumour immunity. Nature 548(7668), 471-475 (2017).

15. Wang B, Niu D, Lai L, Ren EC. P53 increases MHC class I expression by upregulating the endoplasmic reticulum aminopeptidase ERAP1. Nat. Commun. 4, 2359 (2013).

16. Wang X, Sun Q. TP53 mutations, expression and interaction networks in human cancers. Oncotarget 8(1), 624-643 (2017).

17. Wang X, Simon R. Identification of potential synthetic lethal genes to p53 using a computational biology approach. BMC Med. Genomics 6(1), 30 (2013). 
18. Guo J, Pang X, Cheng F et al. Suppression of KRas-mutant cancer through the combined inhibition of KRAS with PLK1 and ROCK. Nat. Commun. 7, 1-13 (2016).

19. Yim H, Erikson RL. PLK1-targeted therapies in TP53- or RAS-mutated cancer. Mutat. Res. Rev. Mutat. Res. 761, 31-39 (2014). 